\title{
Nachgefragt \\ Leitlinien-Empfehlung gilt nur für ein ganz bestimmtes Pflanzenpräparat
}

? Herr Dr. Kardos, was war die Motivation der Deutschen Gesellschaft für Pneumologie, eine S3-Leitlinie Husten $z$ u erstellen?

Kardos: Husten ist das Symptom beinahe aller pneumologischen Erkrankungen. Husten ist die häufigste Beschwerde, die Patienten zum Arzt führt. Verantwortlich für diese extreme Häufigkeit sind akute Erkältungsinfekte und der Husten ist dabei ein besonderes Problem.

? Solche Infekte heilen aber doch meist von allein $a b$, ist eine ärztliche Intervention überhaupt nötig?

Kardos: Da haben Sie Recht, meist würde es reichen, einfach zwei bis drei Wochen abzuwarten. Aber die Patienten kommen ja nicht in die Praxis, um sich zum x-ten Mal die Weisheit anzuhören, "das heilt von allein ab". Die Patienten fühlen sich schlecht, für sie ist der Infekt etwas Schwerwiegendes, auch wenn wir bei der Untersuchung nichts Schlimmes finden.

? Und beim Husten sehen Sie das Hauptproblem?

Kardos: Ja, Husten ist besonders belastend für den Patienten, er hält länger an als die sonstigen Erkältungssymptome. Das kennen wir ja auch von uns selbst, wenn die Symptome ein oder zwei Wochen dauern.

? Es gibt eine ganze Batterie freiverkäuflicher Erkältungsmittel - auch bei akuter Bronchitis. Ist der Arzt also eher für den Bereich Antibiotika zuständig? Kardos: Auf keinen Fall. Leider zeigt die Erfahrung - und es gibt auch Daten dazu -, dass Antibiotika die am häufigsten verordneten Arzneimittel für die akute Erkältung sind - mit allen negativen Konsequenzen. Dabei haben wir es zu $90 \%$ mit viralen Infekten zu tun. Der häufig unnötige Einsatz von Antibiotika führt nicht nur zu Nebenwirkungen, sondern auch zur Resistenzbildung. Damit wird diese Waffe gegen bedrohliche Infekte stumpf. Hinzu kommt, dass es an der Entwicklung neuer Antibiotika hapert.

? Zurück zur Pharmakotherapie. Das Angebot in der Apotheke ist vielfältig, was nützt denn dem Patienten?

Kardos: Ja, es gibt eine Fülle von rezeptfreien Präparaten mit unterschiedlichen Behandlungsprinzipien, seien es Antitussiva, Mukolytika oder andere Präparate wie NSAR, die vielleicht das Allgemeinbefinden bessern. Die meisten kommen aber nicht an den Kern der Sache heran.

\section{? Das heißt?}

Kardos: Wir reden ja über das Hauptproblem Husten und da gibt es eine Gruppe von Pharmaka, bei denen wir einige wenige Studien gefunden haben, die eine Wirksamkeit bei Husten belegen. Wirksamkeit heißt hier: Die Symptome klingen schneller ab. Daher ist es auch unethisch, wenn ein Patient mit Beschwerden kommt, ihm etwas vorzuenthalten, was die Symptomdauer verkürzen und die Symptomschwere lindern kann.

? Worüber sprechen wir hier konkret? Kardos: Über thymianhaltige Kombinationspräparate, mit Efeu oder mit Primel. Auf den ersten Blick mag man es unverständlich finden, dass Thymian sowohl als Antitussivum als auch Sekretolytikum geführt wird. Ich habe mir gesagt, wenn das so ist, dann kann das ja gar nicht wirksam sein. Ich wurde eines besseren belehrt.

\section{? Wodurch?}

Kardos: Ich habe gelernt, dass die Wirkung eines pflanzlichen Arzneimittels von der Herstellungsmethode - zum Beispiel der Art der Extraktion - abhängt. Zwei mittelgroße, placebokontrollierte, randomisierte Studien von Bionorica haben die Wirksamkeit der Kombination belegt. Beim Symptom Husten gab es gegenüber Placebo

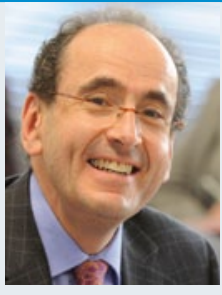

Dr. Peter Kardos

Facharzt für Innere Medizin und Pneumologie, Frankfurt am Main

einen Vorteil von zwei Tagen. Und nach zehn Tagen war der Anteil der beschwerdefreien Patienten mit Verum deutlich höher als mit Placebo. Diese Studien haben sich als so überzeugend erwiesen, dass sie in der evidenzbasierten Leitlinie Husten zu einer starken Empfehlung geführt haben. Heute gibt es meines Erachtens nichts Besseres bei einer akuten Bronchitis, als genau jene Phytopharmaka zu verordnen, die den Beweis der Wirksamkeit erbracht haben.

? Gilt die Empfehlung für Thymianpräparate allgemein?

Kardos: Nein, wir haben ausdrücklich gesagt, dass sich die Empfehlung nur auf einen bestimmten Extrakt bezieht. Es gibt einen wesentlichen Unterschied zwischen chemisch-synthetischen Präparaten und Phytopharmaka: Ambroxol bleibt immer Ambroxol, bei Phytotherapeutika ist das aber nicht so, da kann es keine Generika geben: Die enthaltene Wirkstoffkombination ist abhängig von der Herstellung, sie kann nicht durch die verwendete Pflanze allein chemisch definiert werden.

? Wenn ich Sie recht verstehe, gilt das nur für das Präparat von Bionorica SE, mit dem die Studien gemacht wurden. Um welches Mittel geht es?

Kardos: Das haben Sie richtig verstanden. Es geht um Bronchipret ${ }^{\oplus}$.

? Ein Präparatenamen wird aber in der Leitlinie nicht genannt, warum?

Kardos: Von der Arbeitsgemeinschaft der wissenschaftlich-medizinischen Fachgesellschaften (AWMF) gibt es klare Vorgaben. Und eine davon ist: keine Nennung von Präparatenamen. In der Leitlinie steht jedoch, dass sich die Empfehlung auf einen ganz bestimmten Extrakt bezieht.

! Herr Dr. Kardos, vielen Dank für das Gespräch. 